\title{
Comunicar lo comunitario
}

\section{Communicating communitarian approach}

\author{
Alba Pirla Santamaría \\ Universidad de Lleida \\ apirla@geosoc.udl.cat \\ Ramón JULIÀ TRAVERIA \\ Universidad de Lleida \\ rmjulia@geosoc.udl.cat
}

Recibido: 22/10/2013

Revisado: 09/01/2014

Aceptado: 11/02/2014

Disponible on line: $27 / 06 / 2014$

\begin{abstract}
Resumen
En tiempos de crisis es bueno y necesario reflexionar sobre algunos elementos fundamentales de nuestra profesión y aprovechar la ocasión para poner en relación a los diferentes protagonistas que van construyendo a diario nuestra disciplina. Por un lado, la vertiente académica, que incluye al profesorado responsable de la docencia e investigación, y al alumnado, que es el futuro de la profesión. Por otro lado, al mundo profesional que engloba a los responsables de las administraciones públicas e instituciones sociales que ofrecen servicios sociales y de bienestar social, y a los profesionales que llevan a cabo sus funciones. De la relación entre este conjunto de actores sociales surgen dificultades y potencialidades. Es imprescindible que el Trabajo Social realice un conjunto de planteamientos que conviertan sus amenazas en oportunidades y que nuestra aportación fortalezca la sociedad.
\end{abstract}

Palabras clave: Trabajo Social, comunicación, trabajo comunitario, nuevas tecnologías.

\begin{abstract}
In times of crisis it is good and necessary reflecting on some basic elements of our profession and to take this opportunity to relate the different actors who are constructing our daily discipline. On the one hand, the academic side, this includes professors who are responsible for teaching and research, and students, who are the future of the profession. On the other hand, the professional world comprising the heads of government and social institutions that provides social services and social welfare, and the professionals who carry out their functions. From the relationship between this set of stakeholders arise difficulties and possibilities. It is essential that the Social Work make a set of approaches that turn their threats into opportunities and strengthen our contribution to society.
\end{abstract}

Keywords: Social Work, communication, community service, new technologies.

Referencia normalizada: Pirla Santamaría, A., y Julià Traveria, R. (2014): «Comunicar lo comunitario». Cuadernos de Trabajo Social, 27(1): 139-152.

Sumario: 1. Sobre qué y por qué queremos comunicar. 2. En el ámbito de la formación. 3. En el ejercicio profesional. 4. Algunas preguntas. 5. Algunas propuestas. 6. Referencias bibliográficas.

\section{Sobre qué y por qué queremos comunicar}

En los últimos tiempos sentimos una inquietud creciente, aunque disfrutamos de una profesión versátil, polivalente, y creativa, observamos una tendencia a encajonarnos y a etiquetarnos, y a reducir nuestra aportación social a la de mera gestión de recursos.
Como profesionales del Trabajo Social que somos, sabemos que nuestra misión es mucho más amplia, y como disciplina, poseemos una formación, unas competencias, unos modelos de intervención y una metodología que nos permite realizar intervenciones sociales reales, con el fin de transformar la sociedad. Esto es 
así porque trabajamos tanto a nivel individual, como grupal y comunitario, y somos agentes activos de cambio social, siendo ésta, entre otras, nuestra razón de ser ${ }^{1}$.

Ante esta paradoja nos planteamos inmediatamente varias preguntas. ¿Sabemos vender nuestra profesión en esta sociedad del marketing? ¿Sabemos explicar y transmitir al alumnado de las universidades nuestra profesión? ¿Sabemos ejercer y dar sentido a nuestra profesión cotidianamente? ¿Saben realmente los políticos, gerentes, directores y otros cargos, cuál es la esencia y la misión del Trabajo Social?

Tras este inicio directo y rotundo podríamos pasar directamente a responderlas. No obstante, para hacerlo queremos empezar exponiendo nuestro punto de vista basado en la experiencia. Se trata de nuestro recorrido por el Trabajo Social comunitario, explicando nuestra visión desde diferentes perspectivas y enfoques, presentando algunos argumentos que den respuesta a estas preguntas.

Nuestra experiencia nace, por un lado, de la vertiente teórica y académica como profesores de Grado de la Universidad de Lleida, que impartimos diversas asignaturas, entre ellas: Intervención comunitaria, Modelos de intervención en Trabajo Social o Trabajo Social en atención primaria; todas ellas relacionadas con la intervención comunitaria y, por lo tanto, como conocedores de un ámbito que ha cambiado notablemente durante este periodo. Ha evolucionado tanto en su planteamiento teórico, conceptual y metodológico, como en su implementación real, dependiendo ciertamente de diversos factores socioeconómicos y, por qué no decirlo, con resultados realmente muy dispares $^{2}$.

Por otro lado, nuestras experiencias profesionales, como trabajadores sociales, nos han conducido por diferentes ámbitos, entidades y proyectos que llevan el sello de lo comunitario sin haber sido a menudo muy conscientes de ello. La intervención comunitaria ha estado presente en gran parte de las actividades profesionales que hemos desarrollado, y por este motivo queremos plantear nuestras reflexiones partiendo de la práctica pero también de la teoría y del mundo académico, con el fin de conectar estas dos realidades y poner sobre la mesa temas y debates que en muchas ocasiones nuestra profesión no se plantea o no los quiere debatir de una forma abierta y clara.

\section{En el ámbito de la formación}

En la vertiente académica, el Trabajo Social comunitario suele ser muchas veces ese gran desconocido que aparece en la vida del alumnado de repente, sin demasiadas presentaciones previas. En muchos casos se les ha anunciado en algún curso anterior o en alguna asignatura - con aquella con la que se comparte lo que el nuevo plan de estudios denomina módulo común - pero en muchos casos $l o$ comunitario carece de grandes defensores y seguidores y, por lo general, la publicidad incentivadora es muy baja, cuando no nula o casi nula, salvo algunas excepciones ${ }^{3}$.

Cuando se le plantea al alumnado si conoce lo comunitario su respuesta es normalmente negativa; pero si además se le pregunta si le gustaría trabajar en proyectos de esta naturale$\mathrm{za}$, la respuesta es la indiferencia. Uno de los

${ }^{1}$ Así lo recoge, entre otros, el Código deontológico del Col.legi Oficial de Treball Social de Catalunya en su introducción, que hace referencia a las funciones del Trabajo Social, y en el capítulo 6 a su responsabilidad con la sociedad.

${ }^{2}$ En este sentido encontramos una variedad de experiencias que se han desarrollado al amparo de modalidades de intervención muy dispares, como: los «proyectos de desarrollo comunitario», fruto de los planes de barrio en Cataluña; los «proyectos de desarrollo comunitario e interculturalidad», financiados por la Obra social de La Caixa en toda España; el proyecto «CiutatBeta», impulsado por el Col.legi Oficial d' Educadors i Educadores Socials de Catalunya, que combina el trabajo social comunitario, la acción y gestión sociocultural, la comunicación, las TIC, y toma como protagonista el espacio público y la interdisciplinariedad; y los proyectos innovadores impulsados por Innobasque (Agencia Vasca de Innovación) que favorece e impulsa el desarrollo de la innovación con el objetivo de monitorizar los procesos de transformación, implicando al conjunto de la sociedad.

3 Ésta afirmación se fundamenta en las reflexiones, aportaciones y valoraciones que surgen, en el transcurso de los años, con el alumnado, cuando al inicio de cada curso recogemos los conocimientos previos acerca de la asignatura, las expectativas y las motivaciones de partida. 
motivos por los que creemos que sucede así es por falta de consenso sobre la importancia que se atribuye a la intervención comunitaria, en cualquier tipo de intervención social. Tenemos, entre otras, las aportaciones de Navarro (2004, 2011) que nos abren de par en par una nueva forma de entender el Trabajo Social con otra perspectiva, con otra mirada. De Robertis (2007) también nos lo plantea en sus propuestas de intervención social, a partir de una doble visión: la del individuo versus el colectivo y viceversa, ilustradas por su conocida pirámide invertida. No se trata sólo de conocer estas aportaciones, sino de hacerlas propias e integrarlas en nuestro discurso académico y, posteriormente, en la praxis profesional, de forma progresiva y decidida. Así, la lógica que se sigue planteando en los planes de estudio del Grado de Trabajo Social sigue siendo el fraccionamiento de la intervención profesional en tres niveles: individual, grupal y comunitaria, que el alumnado integra de forma atomizada.

No cabe duda que trabajar en el aula las competencias que requiere una asignatura como la de Intervención comunitaria es difícil. Las habilidades que requiere en la práctica la cuestión grupal, el trabajo en equipo, y experimentar procesos de participación y organización de colectivos es tarea difícil en lo que denominaríamos prácticas de laboratorio. En este sentido, Royo, Sánchez, Lacomba, Marí i Benlloch (2012) plantean precisamente la necesidad de desarrollar proyectos de innovación docente para profundizar en las competencias específicas y necesarias para afrontar precisamente la intervención comunitaria.

Por otra parte, observamos que a menudo la resistencia del alumnado a imaginar y concebir una intervención comunitaria es tan elevada que nos produce a veces la sensación de que estamos justificándonos ante ellos e intentando convencerles de las bondades de lo comunitario. Esto refuerza la idea de que, en su construcción social de la profesión, cuesta mucho de encajar el Trabajo Social comunitario y la visión comunitaria ${ }^{4}$.

A la realidad académica hay que añadirle la dificultad que encontramos para que el alumnado pueda realizar sus prácticas en proyectos comunitarios reales. En este sentido podemos decir que, en estos años de docencia y de supervisión de prácticas, solamente en una ocasión una persona realizó las prácticas en un proyecto específico de desarrollo comunitario $^{5}$. Tampoco ayuda que el alumnado que ya ha realizado prácticas en cualquier ámbito, se reafirme en que el Trabajo Social comunitario no existe o si existe en algunas ocasiones es sólo en los planes, pero no en la realidad ${ }^{6}$.

Sucede así porque no existe una oferta de las instituciones para realizar este tipo de prácticas, ya que no hay de forma explícita este tipo de proyectos, salvo contadas excepciones, y en la mayoría de los casos existe un desconocimiento en las propias entidades de que la labor que están llevando a cabo se puede considerar trabajo social comunitario. Son los mismos profesionales quienes muchas veces no saben

4 Esta dificultad se puede observar por las respuestas del alumnado ante la propuesta de elaborar distintos proyectos comunitarios en el marco de los trabajos grupales tutelados por el profesor.

5 Según datos facilitados por la Unidad de Planificación docente de la Facultad de Ciencias de la Educación de la Universidad de Lleida, en los últimos cinco años, que abarcan desde el curso 2008-2009 al 2012 2013, han realizado prácticas de Trabajo Social 352 alumnos en total, distribuyéndose de la siguiente forma: 200 de Diplomatura en Trabajo Social, 109 de Practicum, 1 de Grado en Trabajo Social y 43 de Practicum, 2 de Grado de Trabajo Social. Dándose solamente un caso de un alumno que realizaba prácticas en un proyecto comunitario de forma específica. Concretamente en el proyecto comunitario del Poble Sec de Barcelona.

Por otra parte, en Lleida se realizan proyectos de desarrollo comunitario desde 2004, ininterrumpidamente, en diferentes barrio y en el marco de los planes de barrio, sin que ningún/una alumno/a haya solicitado realizar las prácticas en esta modalidad de programas, según datos de la misma Unidad de Planificación. Podemos decir que, a pesar de la valoración positiva que realiza el alumnado en las encuestas de opinión, cuando reconocen el valor de esta asignatura para su futuro profesional, pone de manifiesto la dificultad que tiene para apostar por este tipo de trabajo.

6 Esta afirmación se basa en la información obtenida a partir del seguimiento y supervisión del alumnado que ha realizado los diferentes practicum, tanto los de la Diplomatura en Trabajo Social como los de Grado, durante todos estos años en la Universidad de Lleida. 
dar valor a ciertas intervenciones y acciones que realizan, que tienen una vertiente comunitaria clara.

Se da incluso en ocasiones una situación realmente curiosa, cuando un centro, ante la disyuntiva de acomodar a un/a alumno/a en su lugar de prácticas u otro se le aconseja el trabajo individual en vez del grupal o comunitario porque de esta forma aprenderá más y mejor nuestra profesión. Creemos que esta visión también la comparten una gran parte de los profesionales, mujeres y varones, del Trabajo Social.

Por lo que se refiere a las propuestas teóricas elaboradas por diferentes autores entorno al Trabajo Social comunitario en el siglo XXI, encontramos dos grandes líneas de reflexión. Por un lado, el estudio y análisis sociológico de las amenazas y oportunidades que nos brinda el nuevo contexto social para el desarrollo de propuestas y proyectos de tipo comunitario, de autores como Beck (1998), Castells (2001), Bauman (2006), Touraine (2009) o Morin (2010) y, por otro lado, las propuestas metodológicas contrastadas desde la realidad práctica que nos ayudan a desarrollar nuestro trabajo de una forma más ordenada y sistematizada, como Navarro (2004), Malagón (2006), Cortés y Barbero (2006), De Robertis y Pascal (2007) y Fernández y López (2008) entre otros.

En lo referente a lo primero nos encontramos ante un gran reto social en el que las/os trabajadoras/os sociales podemos tener un papel destacado o protagonista, pero requiere a la vez una visión amplia, comunitaria y renovada de la profesión. Estas amenazas y oportunidades las recogemos en el Cuadro 1.
Los y las trabajadoras sociales hemos tenido históricamente la virtud de identificar con facilidad las amenazas, los problemas y las necesidades. En realidad muchas veces nos consideramos expertos en detectar los aspectos negativos, pero en cambio, nos ha costado visualizar las oportunidades y los retos que se nos presentan. El contexto social actual nos invita a replantearnos nuestra misión y nuestra razón de ser. En este cuadro y de forma especial en la columna de las oportunidades se encuentran subyacentes gran parte de las claves y de los proyectos comunitarios que tenemos pendientes y que debemos afrontar con valentía desde esta visión comunitaria, porque desde lo individual parece poco asumible. Como afirma Verde (2008):

El inicio del milenio exigió a los trabajadores sociales $-\mathrm{y}$ les sigue exigiendo- grandes esfuerzos de readaptación tanto teórica como metodológica para adecuarse a las nuevas situaciones sociales, los nuevos contextos de trabajo y las nuevas prácticas profesionales, en los que debe inexcusablemente motivarse, formarse, renovarse y comprometerse éticamente con la justicia social para ser un buen profesional del trabajo social (p. 56).

Existe actualmente en el mundo académico una dificultad añadida. Podemos afirmar de forma nítida que el alumnado, al que debemos explicar este nuevo contexto, pertenece a una nueva generación que nada tiene que ver con las anteriores. Es al mismo tiempo objeto y sujeto del cambio social, es el protagonista de una nueva sociedad que se ha ido generado en los últimos años y, por lo tanto, es difícil reco-

\begin{tabular}{|c|c|c|}
\hline $\begin{array}{l}\text { Conceptos sociológicos } \\
\text { propuestos }\end{array}$ & Amenazas & Oportunidades \\
\hline Sociedad red ${ }^{7}$ & $\begin{array}{l}\text { Exclusión. Culturas contrapuestas y enfren- } \\
\text { tadas }\end{array}$ & $\begin{array}{l}\text { Vinculación -diversidad cultu- } \\
\text { ral-Interculturalidad-redes so- } \\
\text { ciales }\end{array}$ \\
\hline Sociedad del riesgo ${ }^{8}$ & $\begin{array}{l}\text { Miedo a lo diferente -cierre incertidumbre- } \\
\text { discriminación-racismo }\end{array}$ & Confiar en los otros-solidaridad \\
\hline
\end{tabular}

${ }^{7}$ Concepto propuesto por Manuel Castells y que se ha convertido en una referencia indiscutible para cualquier análisis de la sociedad actual en el que las nuevas tecnologías se han convertido en el motor de cambio social.

${ }^{8}$ Idea elaborada por Ulrich Beck que adquiere una gran relevancia en los momentos actuales y que resume, en pocas palabras, el sentir de toda una sociedad en momentos de crisis y de cambio social como el actual. 


\begin{tabular}{|c|c|c|}
\hline $\begin{array}{l}\text { Conceptos sociológicos } \\
\text { propuestos }\end{array}$ & Amenazas & Oportunidades \\
\hline $\begin{array}{l}\text { Sociedad programa- } \\
\mathrm{da}^{9}\end{array}$ & $\begin{array}{l}\text { La sociedad como objeto y Rigidez en los } \\
\text { programas }\end{array}$ & $\begin{array}{l}\text { Recuperación del sujeto social y } \\
\text { uso de la racionalidad }\end{array}$ \\
\hline Sociedad compleja ${ }^{10}$ & Simplificación del pensamiento-rigidez & $\begin{array}{l}\text { Uso del pensamiento complejo- } \\
\text { flexibilidad estrategia de acción }\end{array}$ \\
\hline $\begin{array}{l}\text { Sociedad seculariza- } \\
\mathrm{da}^{11}\end{array}$ & $\begin{array}{l}\text { Nuevas formas de religiosidad-cambio de } \\
\text { valores-rechazo hacia la religión }\end{array}$ & $\begin{array}{l}\text { Pluralismo religioso. Nueva es- } \\
\text { piritualidad }\end{array}$ \\
\hline $\begin{array}{l}\text { Sociedad individua- } \\
\text { lizada }^{12}\end{array}$ & $\begin{array}{l}\text { Aislamiento del individuo-individualismo } \\
\text { irresponsable-anomia social-analfabetismo } \\
\text { relacional }\end{array}$ & $\begin{array}{l}\text { Libertad individual. Individua- } \\
\text { lismo responsable. Recupera- } \\
\text { ción valores colectivos }\end{array}$ \\
\hline $\begin{array}{l}\text { Sociedad de la in- } \\
\text { formación }^{13}\end{array}$ & $\begin{array}{l}\text { Censura por sobreinformación, manipula- } \\
\text { ción }\end{array}$ & $\begin{array}{l}\text { Acceso a la información en un } \\
\text { mundo globalizado } \\
\text { Sociedad del conocimiento } \\
\text { Ética informacional }\end{array}$ \\
\hline $\begin{array}{l}\text { Sociedad del espec- } \\
\text { táculo }\end{array}$ & $\begin{array}{l}\text { La vida como un plató de televisión. Hiper- } \\
\text { emotividad }\end{array}$ & $\begin{array}{l}\text { El espectáculo como herramien- } \\
\text { ta educativa }\end{array}$ \\
\hline Cibersociedad $^{15}$ & $\begin{array}{l}\text { La dificultad para las relaciones sociales ca- } \\
\text { ra a cara }\end{array}$ & La creación de redes sociales \\
\hline Sociedad líquida ${ }^{16}$ & $\begin{array}{l}\text { Dificultad para identificar aquello que antes } \\
\text { era claro y estable }\end{array}$ & $\begin{array}{l}\text { Dinamismo en las estructuras y } \\
\text { relaciones }\end{array}$ \\
\hline
\end{tabular}

Cuadro 1. Sinopsis de la perspectiva profesional comunitaria.

Fuente: Elaboración propia.

nocer y ser críticos sobre aquello en lo que participa y comparte desde la infancia. El ejemplo más claro lo encontramos en el uso y la incorporación de las nuevas tecnologías a su vida cotidiana, que se ha convertido, como planteaba Sartori (2002), en una arma de doble filo para su aprendizaje, su capacidad de abstracción, de esfuerzo, de creatividad y de análisis

${ }^{9}$ Concepto planteado por Alain Touraine, de forma muy crítica, para referirse al modelo social que se ha construido en los últimos tiempos, se basa en la racionalización y se debe replantear para la búsqueda de un nuevo paradigma que ofrezca respuestas más adecuadas a las nuevas realidades sociales centradas en el sujeto.

10 Planteamiento que surge de la aportación de Edgar Morin en su obra y que pone énfasis en la ruptura que se ha producido entre el paradigma científico de las ciencias naturales y el paradigma humanista de las ciencias sociales, y que ha provocado un alejamiento que divide a la sociedad y resta responsabilidad a cada una de las partes, en vez de añadir y sumar esfuerzos para alcanzar una mejora social.

${ }^{11}$ Concepto de gran relevancia en contextos multiculturales como los actuales, así como en aquellos en los que la interculturalidad se plantea como un reto, y la religión puede ejercer un papel protagonista.

${ }_{12}$ Concepto que surge del análisis profundo del individualismo que realiza Gilles Lipovetsky a lo largo de su obra y que pone de manifiesto su doble cara del individualismo y la necesidad de reflexionar sobre sus causas y sus consecuencias.

${ }^{13}$ Concepto propuesto también por Manuel Castells, para reforzar la idea de la sociedad que surge después de la gran revolución que ha supuesto la aparición de las nuevas tecnologías y la sociedad red.

${ }^{14}$ Concepto elaborado por Joan Ferrés, en relación a los retos de la educación en una sociedad en la que se impone el espectáculo en todas sus facetas y de cómo incorporarlo de forma positiva a la educación.

${ }_{15}$ Concepto utilizado por Dominique Wolton, entre otros autores, para referirse a la influencia de las nuevas tecnologías de la información en la sociedad contemporánea.

${ }^{16}$ Zygmunt Bauman es el autor que ha desarrollado el concepto de sociedad y de modernidad líquidas a lo largo de su obra y nos invita a la reflexión sobre los cambios en nuestra sociedad en lo que se refiere a su estructura social, las relaciones sociales, las organizaciones etc. 
de la realidad, eso sí, siempre bajo la mirada de nuestra generación y de las anteriores.

En lo referente al segundo aspecto, el relativo a las aportaciones sobre metodologías y procesos, cabe destacar las de Barbero y Cortés (2005), Malagón y Sarasola (2006) y Fernández y López (2008) que, siguiendo en gran parte la línea iniciada por Marchioni (1987), actualizan y sintetizan de forma clara los aspectos más relevantes para una intervención comunitaria actual. Reconociendo de antemano su valor, creemos que es necesario recordar con la misma intensidad la importancia de los modelos de intervención en el Trabajo Social, que son los que realmente y en última instancia dan sentido a la práctica, como señalaba Howe (1999).

Otro de los grandes debates teóricos que se presenta en el trasfondo de la cuestión es precisamente el significado de «comunidad». Ha sido, y creemos que sigue siendo, el gran caballo de batalla de nuestra profesión. Como ya recogían Villalba (1993), Villasante (1999), Lillo i Rosselló (2001), Navarro (2004bis) y Pastor (2004), hay que plantear conceptos alternativos al de comunidad, como por ejemplo: el de colectivo, el de red social o el de barrio, pero al final siempre acaba resonando el concepto «comunitario».

En este sentido existen diferentes propuestas, reflexiones y enfoques que nos permiten vislumbrar la complejidad de este debate. Por un lado, encontramos las amenazas a las que nos enfrentamos en tiempos de crisis y que generan lo que Touraine (2009) y Bauman (2006) llaman comunitarismos, y que, por ejemplo, en el caso de la integración de las personas inmigradas en nuestra sociedad, nos encontramos con que muchas veces la comunidad, y cierta concepción rígida y tradicionalista de la comunidad y del comunitarismo, han sido precisamente los factores que han dificultado esta integración. Estos autores avisan de su peligro en tiempo de incertidumbre y de inseguridad y que su atractivo es precisamente la promesa de un refugio seguro ante los cambios constantes en los tiempos actuales.

Otros autores han pecado, según Hernández (2009), de tener una visión excesivamente paradisíaca de este concepto como por ejemplo Tönnies, en su ya clásica distinción entre comunidad y sociedad. Malagón (1989) hablaba de la idealización de la comunidad, precisamente por el carácter polisémico del propio concepto y las connotaciones a ella asociadas.

Para Saraceno (2008), en el ámbito de la salud mental es una cuestión que debe plantearse de forma decidida; y se pregunta ¿por qué seguimos hablando de comunidad como si se tratara de un contexto semirural cuando realmente nos encontramos en contextos urbanos violentos? Nos encontramos ante sufrimientos complejos y no lineales, grupales y no individuales, y por lo tanto hay que buscar respuestas complejas y grupales.

Por otro lado, existen valoraciones y reflexiones que devuelven el sentido y el valor de la comunidad, aunque asumiendo nuevos planteamientos conceptuales sobre la misma.

Para Malagón (2006) la comunidad es fundamental porque precisamente muchas veces las actitudes racistas nacen y se desarrollan en su interior, siendo por lo tanto el lugar más adecuado para realizar trabajo social, para evitar y prevenir estas situaciones.

Sánchez, Escobar y Andrés, por su parte (2002), plantean la comunidad como una gran apuesta para fabricar culturas inclusivas. Por otra parte, Castells (2001) nos advierte de que las redes sociales han sustituido a las comunidades y que las nuevas tecnologías se presentan como una gran oportunidad en este nuevo contexto. Estas redes, según Bott (1992), ya no tienen necesariamente, como referente espacial o territorial, el lugar de residencia.

Queda claro que en el mundo académico tenemos un gran reto ante la responsabilidad y misión de transmitir al alumnado el significado de la comunidad en los tiempos actuales, para que puedan visualizar de la forma más clara, reconocible y asumible, el ámbito de intervención comunitaria que se les propone. Como plantea Brake (2008),

Se hace evidente que falta rediseñar las comunidades locales como lugares de vida con proyección de futuro, y capacitar a las personas para recuperar su competencia de acción individual. Se trata de recuperar y revivir recursos locales perdidos en el proceso de globalización (p. 97).

\section{En el ejercicio profesional}

Queremos iniciar este apartado repasando brevemente las diferentes experiencias de trabajo comunitario, en el sentido más amplio de su 
acepción, en las que hemos podido participar durante nuestra trayectoria profesional. Por un lado, en la coordinación de proyectos de voluntariado en entidades del Tercer Sector, en proyectos comunitarios EQUAL ${ }^{17}$, en la creación, planificación y elaboración de proyectos de desarrollo rural en el marco de las iniciativas Leader $^{18}$, en proyectos innovadores en el marco de la ocupación, trabajando en red con entidades sociales, en la organización de planes de barrio, en el plan local de inclusión, plan integral sobre el trabajo sexual y en proyectos grupales impulsados desde Servicios Sociales de Atención primaria, como experiencias más significativas; así como en proyectos de responsabilidad social empresarial (RSE), a partir de la dinamización de empresas privadas con el fin de desarrollar estrategias para conseguir un territorio socialmente responsable.

De todas estas experiencias podemos extraer una serie de reflexiones básicas con el ánimo de plantear algunas de las cuestiones que, a nuestro entender, han dificultado una mejor implementación de los proyectos.

En primer lugar, creemos que en los últimos años se ha dado mucha importancia a la metodología y a la sistematización de las intervenciones, pero no tanta a los modelos que acompañan a las acciones; es decir, qué teorías, qué valores y qué principios mueven estos proyectos ${ }^{19}$. Esta forma de proceder ha conllevado un tipo de interven- ción comunitaria muy determinada y de alguna forma estandarizada y poco singularizada. Así mismo, se ha priorizado la gestión y la organización sobre la construcción o la revisión de modelos teóricos-ideológicos y prácticos.

En segundo lugar, creemos que, en muchas ocasiones, los objetivos de la acción en los proyectos comunitarios han estado demasiado pendientes de los denominados objetivos de tarea, es decir, más atentos a los resultados finales y la visibilidad y vistosidad de las acciones, que preocupados por los objetivos llamados de proceso, que son los que ponen el énfasis en las relaciones y las dinámicas grupales que se establecen, los compromisos y la implicación cotidiana de los ciudadanos ${ }^{20}$. Los ritmos e intereses políticos y presupuestarios han dejado atrás al de los procesos comunitarios, acelerando resultados y respetando poco los tempi comunitarios, obteniendo resultados óptimos a nivel de satisfacción institucional, pero poco valorados por los mismos profesionales y por la comunidad en muchos casos receptora de acciones y no cómplice y co-constructora.

Estamos de acuerdo con aquella frase que reza «si no hay organización no hay participación» pero hay que creer ciegamente en la participación para que sea auténtica y hay que tener al mismo tiempo un nivel de flexibilidad suficiente como para no caer en las garras de la rigidez y la burocracia organizativa.

17 Los proyectos EQUAL debían regirse por un conjunto de principios básicos como: la cooperación institucional y la capacitación, la innovación, la complementariedad, la igualdad de oportunidades, la capacidad de transferencia, el enfoque temático e integrado, la concentración y la cooperación trasnacional. Todos ellos son también propios de cualquier intervención comunitaria.

18 La iniciativa comunitaria LEADER así como la EQUAL, planteaba una metodología basada en los principios básicos de una intervención comunitaria, en este caso aplicados al desarrollo rural. «El método LEADER se caracteriza por siete aspectos esenciales: el enfoque territorial, el enfoque ascendente, la creación de un partenariado, el carácter innovador de sus acciones, el enfoque integrado (multisectorial), la introducción en red y cooperación, y la gestión y financiación de proximidad» (AEIDL, 1999).

19 Jaraiz (2012) plantea esta cuestión a partir de un estudio realizado por la Universidad Pablo Olavide de Sevilla, para conocer el estudio de las prácticas y discursos de los profesionales de Servicios Sociales comunitarios acerca del sentido de su intervención. El autor expone en diferentes ocasiones esta realidad y concluye: «En los Servicios Sociales objeto de este estudio ha primado un modelo de intervención asentado en prácticas en las que las comunidades trataban como objeto sobre el que aplicar acciones de naturaleza facilitadora; relegándose a un segundo plano la intervención de lógica empoderadora y activadora, que concibe al barrio y sus vecinos como sujetos del propio proceso de acceso al bienestar...»( p. 123)

20 Cortés (2003), en un estudio de aproximación a los planes comunitarios desarrollados en Cataluña en los últimos años, plantea una hipótesis que refuerza esta misma idea: «Los planes comunitarios muchas veces quedan limitados a plataformas de coordinación de entidades privadas que existen en el territorio, que sirven para fomentar el trabajo en común entre estas entidades, y sobre todo para ordenar las subvenciones que estas reciben de las administraciones públicas y quedan, por lo tanto muy lejos del plan comunitario ideal que define Marchioni (p.24). 
Se predica que hay que implicar a la ciudadanía, pero nos queda la duda de si cuando hablamos de ciudadanos/as incluimos a todas aquellas personas afectadas, que son en realidad las verdaderas protagonistas de sus problemas y necesidades. Sin olvidar algunas contradicciones como la existencia de una gran parte de la población residente en nuestro país que no gozan del status de ciudadanos plenos, pero que conviven cotidianamente con nosotros.

Las administraciones públicas han invertido grandes cantidades de dinero en proyectos de desarrollo comunitario en el marco de los planes de barrio, como ha sido el caso de Catalunya. Estos proyectos han dinamizado los barrios y han llevado a cabo actividades por doquier durante largo tiempo. Se produce, sin embargo, una gran paradoja. ¿Qué queda?, ¿qué permanece una vez finalizados? En muchos casos sólo quedan y permanecen las acciones urbanísticas y las infraestructuras que se hayan podido crear. Del bienestar social, de la calidad de vida, de la cohesión social, de la participación, de la interculturalidad no sabemos nada. No se visualiza ${ }^{21}$. Y lo más preocupante, a nuestro parecer: ni siquiera se evalúa su impacto sobre los niveles de calidad de vida de sus ciudadanos. Como dice Jover (2010), lo que no se evalúa se devalúa.

En muchos casos, los proyectos los han llevado a cabo equipos creados especialmente para este fin y sin que tuvieran una conexión directa con los Servicios Sociales de Atención primaria o similares. A menudo, se han desarrollado actividades que giraban en torno a la inserción laboral como la única vía para la inserción e integración sociales, descuidando otros aspectos dirigidos a la cohesión social o a la interculturalidad, por poner algunos ejemplos.

Por otra parte, hemos podido observar como muchos proyectos comunitarios, que podrían liderarlos trabajadores/as sociales, no los han tenido en cuenta y se han buscado otros perfiles que nada tienen que ver con lo social, o al menos con el profesional del Trabajo Social. Lo hemos podido constatar especialmente en proyectos de desarrollo económico y comunitario a nivel local, en proyectos de desarrollo rural y en proyectos de fomento de la ocupación, entre otros. El perfil del trabajador/a social no se ha considerado idóneo, en muchas ocasiones, precisamente por su identificación con la exclusión, marginación, trabajo individual y gestión de recursos.

La dimensión social de muchos de estos proyectos se ha considerado innecesaria o secundaria en bastantes ocasiones, aunque se tratara de uno de los principios transversales de todos ellos. En definitiva, se ha apostado por perfiles procedentes del ámbito empresarial o similar, en vez de por perfiles del ámbito social. Se ha pensado más en la gestión que en la población.

Por otra parte, el empoderamiento se ha convertido a nivel teórico en el gran objetivo final de una gran cantidad de proyectos de diferente índole. El empowerment es la palabra más usada en los últimos años, como la gran solución a nuestros males; pero desde nuestro punto de vista, el empoderamiento debe ser un principio básico, una premisa, un posicionamiento desde el inicio de la acción, no el objetivo final como se plantea en la mayoría de los casos. Debemos añadir a este punto la sensible diferencia existente entre habilitar a la población y/o empoderarla, como plantea Pastor (2004).

En los tiempos que corren, en Trabajo Social no podemos limitarnos, ni se nos puede limitar, a querer organizar a la comunidad. Por otra parte, es imprescindible ahondar en la concienciación social junto a la sensibilización y el fomento de la solidaridad, aunque ya sabemos que es un trabajo constante y de largo alcance.

21 Vecina y Ballester (2012), refiriéndose a proyectos desarrollados en la Islas Baleares, plantean el peligro de esta situación y realizan la siguiente reflexión: «Consideramos que no pueden pensarse rehabilitaciones urbanísticas sin integrar proyectos de dinamización social que incluyan sus inquietudes y necesidades en el proceso. Aunque su avance a diferentes ritmos obliga a adaptar las intervenciones, en nuestro caso a fomentar unas u otras comisiones de trabajo. El apoyo de las políticas locales es fundamental, pero no puede ser efímero y desaparecer antes de que los procesos de organización social se hayan consolidado. El resultado es una situación de incertidumbre por parte del tejido asociativo y la ciudadanía implicada en las acciones, pues desaparece la figura que ha intervenido, sin que todavía existan líderes o intereses considerados lo suficientemente importantes para reorganizarse y continuar (p. 411). 
Sobre esta cuestión se plantean en la actualidad algunas limitaciones que es necesario apuntar. Por un lado, la evidencia de que la sociedad y los colectivos sólo se mueven en aquellos casos en los que las amenazas son directas y realmente propias, como ha sido el caso de los afectados por la hipoteca. Por otra parte, la impotencia de la sociedad ante una reforma laboral, que, aun existiendo una enorme concienciación social, por decisión unilateral de los gobernantes han impedido cualquier posibilidad de modificación o rectificación de la misma, con la consecuente desmoralización, desmotivación y desilusión social.

Por último, en lo referente a la concienciación, hay que hablar de la manipulación a la que estamos siendo sometidos, nosotros y la sociedad en general, por los gobernantes en base a esta misma concienciación social, sensibilización e interpelación a la solidaridad para responsabilizar a la sociedad del destino de las personas más vulnerables y en riesgo de exclusión social. El resultado de esta estrategia y sus consecuencias son el retorno de la caridad y la beneficencia, como respuesta de una sociedad solidaria con los más pobres, es decir, mayor responsabilidad de la sociedad civil y una desresponsabilización cada vez mayor de las administraciones públicas ante la desigualdad e injusticia social.

Analizando esta situación, con cierta distancia y objetividad, llegamos a la conclusión de que hemos estado desenvolviéndonos entre cantos de sirenas y encantadores de serpientes que nos han sumergido en un letargo del que debemos despertar inmediatamente, si queremos enderezar el rumbo de nuestra profesión y trabajar realmente por la igualdad y la justicia social.

En nuestra experiencia práctica en el marco de proyectos comunitarios también hemos podido constatar la gran dificultad, para muchos profesionales de lo social, de afrontar la realidad con perspectivas más amplias, y dejar de centrarse de forma exclusiva en el trabajo individual. En ocasiones es el exceso de carga asistencial, en otras los límites de la institución, pero en casi todas, es la comodidad y la falta de motivación para afrontar retos que supongan un compromiso más profundo con la profesión y con la ciudadanía, que sufre cada vez más la desigualdad e injusticia social.
En un contexto de profunda crisis, es necesario, imprescindible e inevitable recordar la misión del Trabajo Social como lucha por los derechos humanos. También es necesario hablar de ética profesional y de compromiso, para poder realmente regenerar también nuestra profesión.

\section{Algunas preguntas}

Un vez, llegados a este punto, sólo queda responder a las preguntas iniciales, más allá de los argumentos presentados a lo largo del artículo y que, vistos desde una experiencia concreta, han transitado por diferentes aspectos que dan una respuesta y posicionamiento claro de los autores.

\subsection{Sobre si sabemos vender nuestra profe- sión en esta sociedad de marketing total}

Nuestra respuesta es: no. Estamos muy lejos de saber aplicar fórmulas de marketing social a nuestra profesión. Estamos ante uno de los mayores retos actuales y debemos dedicar tiempo y formación en esta dirección, sin pensar por ello que estamos entrando en el juego del consumismo y del mercadeo del capitalismo. Más allá del trabajo que deben realizar las universidades y los colegios profesionales, cada profesional debe y puede convertirse en altavoz y ejemplo de buenas prácticas en su quehacer cotidiano y reivindicar la necesidad de innovar y contar con la comunidad, para sobreponerse (sobreponernos) a la realidad actual.

También en este sentido, es necesaria la construcción de un relato colectivo, que aglutine los discursos individuales, revalorice el presente y permita reconstruir la profesión, adecuándola a los nuevos retos sociales. Más allá de ser en lo individual agentes activos, críticos, autocríticos y comprometidos, y arriesgando hacia el cambio, es necesario construir complicidades, discurso y acción colectiva.

\subsection{Sobre si sabemos explicar y transmitir nuestra profesión al alumnado universitario} Creemos que el profesorado universitario tiene una gran responsabilidad ante este reto y por ello es cada vez más imprescindible el trabajo en equipo, no sólo para realizar proyectos e investigaciones, sino también para definir criterios y ofrecer al alumnado una visión de la profesión real, abierta, positiva y motivadora. Es 
necesario trabajar, en los equipos de docentes de las universidades, de forma transversal, contando con los y las profesionales que se encuentran en el mundo laboral. Si el mundo académico se vincula con el mundo de la práctica profesional pueden surgir intercambios, vínculos, propuestas, metodologías, proyectos, etc., solo es necesario invitar a la co-creación.

\subsection{Sobre si sabemos ejercer y dar sentido} a nuestra profesión en el quehacer cotidiano

Los y las profesionales están cada vez más superados por las circunstancias y por la mayor exigencia y presión asistencial, que genera una desmotivación y angustia creciente. Ante un panorama como este es difícil dar el sentido adecuado a la profesión desde la visión de los últimos años. Es obligación de las administraciones, entidades e instituciones, apoyar y escuchar a sus profesionales en momentos como éste. La mala praxis profesional corre el riesgo de extenderse con el peligro que supone en primera instancia para las personas atendidas pero también en la opinión pública que se pueda generar alrededor de nuestra profesión.

Es también a nuestro entender obligación de los y las profesionales reorientar nuestra práctica y mirada profesional y elevar nuestras inquietudes y propuestas a las instituciones para las que trabajamos.

\subsection{Sobre si saben realmente la clase políti-} ca, los gerentes, directores y demás cargos, cuál es la esencia y misión del Trabajo Social

En este caso la conclusión viene dada por la realidad actual en la que vivimos. Ha quedado demostrado que, aunque los políticos sepan cuál es nuestra misión, generalmente priman intereses políticos y partidistas sobre las necesidades sociales reales. Se da el caso muchas veces de que, aunque una de estas personas con cargos importantes proponga y defienda una idea interesante, siempre acaba prevaleciendo el interés particular por encima del general. Lamentablemente, en estos momentos sobran ejemplos de la dinámica expuesta.

Cuando los políticos, a través de la Administración, pasan de promotores, patrocinadores, socios de proyectos comunitarios, que debían conseguir la cohesión social y el empoderamiento, a convertirse en el principal enemigo, cuando actúa, a partir de recortes en los derechos sociales, sólo nos queda la acción, actuar para conseguir obtener cuotas de poder que nos permitan modificar la realidad desde dentro. ¿Debemos esperar a que sea la clase política la que nos devuelva la ilusión y los derechos?

\section{Algunas propuestas}

Creemos que es necesario redescubrir nuestra profesión para conseguir hacer de la necesidad virtud, aprovechando el contexto para provocar un cambio de modelo. Hay que buscar nuevos paradigmas para afrontar estos nuevos retos.

1) Renovar la práctica a partir de nuevas $y$ creativas oportunidades. Es necesario una reflexión desde dentro, por los mismos profesionales, una apertura hacia la comunidad, una reacción ante la situación que aporte nuevos modelos y construcciones metodológicas. En este sentido es imprescindible generar espacios de autoevaluación de equipos, de instituciones, plantear necesidades y proponer estrategias. Será necesario articular programas de formación que faciliten estos procesos y motiven a los y las profesionales al cambio de paradigma. En este punto, la vinculación del mundo académico con el profesional se convierte en imprescindible. A través de la colaboración bidireccional se pueden promover cambios importantes.

2) Aprovechar las nuevas tecnologías y especialmente el entorno 2.0 para adaptarnos a la nueva realidad social y usarlas como herramienta de creación y no únicamente de consumo.

Hay que creer en los demás y en sus capacidades de forma clara y compartir las experiencias para que la transferencia de conocimientos sea una realidad y no querer poseer las patentes y el copyright de todo aquello que creamos para aumentar nuestro ego por encima del bien común. En este sentido, las redes sociales resultan un claro potencial al servicio de los profesionales, ya que la posibilidad de compartir conocimientos, a través del mundo 2.0, como las redes de contacto profesional, los blogs, webs que atractivamente visualizan proyectos de impacto social, etc., permiten la creación de nuevas ideas a partir de experiencias reales. Son clave en la innovación social y en el desarrollo de iniciativas comunitarias, mediante la 
trasferencia y adaptación a las distintas realidades, a través de las grandes sinergias entre profesionales que nos proporciona el mundo virtual.

Claramente, en un mundo globalizado, disponer de acceso a la información a través de las redes sociales debe verse como una oportunidad de desarrollo profesional, y por continuidad, de desarrollo social partiendo de sus acciones, de la propia aportación profesional, de la inteligencia colectiva, etc. La cibersociedad permite dar un salto a nuevas formas de relación, de co-creación, y a un mayor dinamismo relacional.

Albaigés (2012) y Sánchez (2013), plantean nuevas posibilidades a diferentes niveles, ya sea a través de plataformas que permiten promover la participación, mediante comunidades virtuales, y a su vez compartir y distribuir conocimiento, detectar y construir complicidades intra y extrasectoriales, contribuyendo a la creación de conciencia global, o sea, a la construcción de La Comunidad. Así, las TIC están creciendo y sirviendo de altavoz para los dos discursos: el académico y el profesional, dos discursos que se retroalimentan y que conjuntamente tejen una red de voces hasta ahora impensable.

Creemos que la innovación en el trabajo social comunitario debe incorporar, sin lugar a dudas, nuevos conceptos, nuevas metodologías, creatividad en el hacer y en cómo hacer, y el mundo virtual está a nuestro servicio.

3) Recuperar el modelo de acción social como vía real de empoderamiento. Algunos ejemplos actuales, surgidos de movimientos ciudadanos — como la plataforma Afectados por la hipoteca - pueden ser un referente para los que trabajamos en el ámbito social y alardeamos de querer cambiar y transformar la sociedad. Creemos que existe una manipulación sobre el concepto de poder por su interpretación negativa y casi corrupta. Pero en términos democráticos, es una mayor representación, participación y corresponsabilidad con la vida social y pública. Es necesario devolver a la comunidad su papel protagonista en el desarrollo del guión, fomentando y poniendo en valor estructuras horizontales donde el individuo y la comunidad tejan respuestas colectivas que satisfagan sus necesidades.
El empoderamiento es una de las claves para el cambio social, donde las personas adquieren habilidades de superación, de aprendizaje, de toma de decisiones. El modelo de acción social aporta la visión de la capacidad de las comunidades para movilizarse y resistir contra las adversidades y luchar contra la injusticia social «desde dentro», desde las propias capacidades.

4) Dinamizar nuevas formas complementarias de participación y activismo que respondan a las realidades del siglo XXI.

Así, nuevas formas de participación y activismo, que pueden también tener incidencia en las redes sociales, en cuanto a transmisión de información y fuente de intercambio, son acciones para valorar y promover, incrementando el nivel de participación de los propios afectados por el contexto socioeconómico en la creación de un sistema más justo, mediante la reivindicación y el compromiso social.

Tal y como plantea Sánchez (2013), las redes sociales están también al servicio del ciudadano en estas nuevas formas de acción social, para dimensionar acciones, difundirlas, generar opinión crítica y visibilizar determinadas realidades que de otra forma seguirían siendo invisibles. Así, tenemos el reto de empoderar a la ciudadanía a través del uso de las TIC, denunciar situaciones de desventaja social, formarse online, etc. En definitiva, para mejorar su calidad de vida. Así, se amplía el concepto de Comunidad a través de la creación de comunidades virtuales que den voz a estas personas.

5) Provocar un cambio de percepción des del mundo académico: así, consideramos clave la transversalidad de materias como el trabajo social comunitario. Es necesario introducir de forma clara y activa la visión comunitaria en nuestra construcción social de la profesión para promover una nueva generación de trabajadores/as sociales implicados con la sociedad en el sentido más amplio, que incorporen como un contínuum los distintos niveles de acción social ahora fraccionados.

Es necesaria también la búsqueda de proyectos comunitarios para el desarrollo de prác- 
ticas universitarias. Es imprescindible mejorar las estrategias de prospección de puestos de prácticas específicas para el alumnado de Grado de Trabajo Social con el fin de disponer de un catálogo de lugares en los que se trabaja lo comunitario a diario.

Además el uso de las nuevas tecnologías en las metodologías de las asignaturas nos permite sacar el máximo partido de los campos virtuales que se nos ofrecen pero que muchas veces infrautilizamos por desconocimiento.

Recordar en última instancia que, en los últimos tiempos, asistimos de forma continuada a respuestas colectivas a necesidades individuales y sociales en algunos sectores que en definitiva son contextos de acción social comunitaria que el alumnado conoce y que en algunos casos además están vinculados y ejercen complicidad conjuntamente con profesionales del mundo social (algunos ejemplos, como las Mareas Naranja). Creemos necesario alimentar desde el entorno académico el espíritu y la ac- titud crítica, positiva y de posicionamiento ya desde la cuna de la formación en clave de movilización y participación social.

6) Mayor implicación del trabajador/a social en nuevos ámbitos o recuperar aquellos en que habia presencia anteriormente, como el empleo o la vivienda, entre otros. Las problemáticas sociales varían con el tiempo y del mismo modo que la inserción laboral ha marcado una etapa en el devenir de las entidades sociales, hoy en día se abren nuevos frentes a los cuales hay que dar respuesta, más allá de cubrir necesidades básicas.

La apertura del/de la trabajador/a social a otros ámbitos que no eran propios, como el desarrollo económico y comunitario, con el fin de aportar la visión social, permite apostar por la vertiente polivalente de trabajador/a social comunitario como especialista para el presente y futuro de la profesión.

\section{Referencias bibliográficas}

AEIDL. (Asociación Europea de Información sobre el Desarrollo Local). (1999). Evaluar el valor añadido del enfoque LEADER. Recuperado de: http://superdoc.aeidl.eu/documents/ PUBLICATIONS_AEIDL/LEADER_added-value-ES.pdf

Bauman, Zygmunt; (2006): Modernidad Líquida. Fondo de cultura económica. Buenos aires.

Berger, Peter y Luckmann, Thomas (2001). La construcción social de la realidad. Buenos Aires: Amorrortu Editores.

Beck, Ulrich (1998). La sociedad del riesgo. Hacia una nueva modernidad. Barcelona: Editorial Paidos.

Bott, Elizabetth (1992). Familia y red social. Madrid: Editorial Taurus.

Brake, Roland (2008). Trabajo social comunitario (TSC). En Jesús Hernández Aristu. (comp.), Trabajo social comunitario en la sociedad individualizada.Valencia: Nau Llibres.

Castells, Manuel (2001). La era de la información: La Sociedad Red, I. Madrid: Alianza Editorial

Castells, Manuel (2001). Economía Sociedad y cultura. El poder de la identidad, II. . Madrid: Alianza Editorial

Castells, Manuel (2001). Fin del Milenio.III. Madrid: Alianza Editorial.

Cortés, Ferran y Barbero, Josep Manuel (2006). Trabajo comunitario, organización y desarrollo social. Madrid: Alianza Editorial.

Cortés, Ferran (2003). Una aproximació als plans comunitaris: una manera d'organitzar la comunitat per a promoure processos de desenvolupament social. Revista de Treball Social, 172, 6-40.

De Robertis, Cristina y Henri, Pascal (2007). La intervención collectiva en Trabajo social. La acción con grupos y comunidades. Buenos Aires: Editorial Lumen.

Fernández, Tomás y López, Antonio. (2008). Trabajo social comunitario: afrontando juntos los desafios del siglo XXI. Madrid: Alianza Editorial.

Ferrés, Joan (2000). Educar en una cultura del espectáculo. Barcelona: Ediciones Paidós Ibérica, S.A.

Hernández, Jesús (2009). Trabajo social comunitario en la sociedad individualizada. Valencia: Nau Llibres. 
Howe, David (1999). Dando sentido a la práctica. Una introducción a la teoría del trabajo social. Granada: Editorial Maristán.

Jaraiz, Germán (2012). La «dimensión comunitaria» en Servicios Sociales. Una aproximación a su tratamiento en barrios vulnerables. Cuadernos de Trabajo Social, 25 (1), 113-124.

Lillo, Nieves y Roselló, Elena (2001). Manual para el trabajo social comunitario. Madrid: Ediciones Narcea.

Lipovetsky, Gilles (1996). La era del vacío. Barcelona: Editorial Anagrama.

Lipovetsky, Gilles (2003). Metamorfosis de la cultura liberal. Ética, medios de comunicación, empresa. Barcelona: Editorial Anagrama.

Malagón, José Luís (1989). La idealización de la comunidad. Revista de Treball Social, 116, 11-22.

Malagón, José Luís y Sarasola, José Luis (2006). Fundamentos del Trabajo social comunitario: Bases teóricas y metodológicas para la intervención comunitaria. Sevilla: Aconcagua libros, S.L.

Marchioni, M. (1987). Planificación social y organización de la comunidad. Alternativas avanzadas a la crisis. Madrid: Popular.

Morin, Edgar; (1990): Introducción al pensamiento complejo. Barcelona: Editorial Gedisa.

Morin, Edgar (2006). Cultura i Barbàrie d'Europa. Lleida: Pagès Editors.

Morin, Edgar (2010). La mente bien ordenada: repensar la reforma, reformar el pensamiento. Barcelona: Editorial Seix Barral.

Navarro, Silvia (2004). Redes sociales y construcción comunitaria. Creando (con)textos para una acción social ecológica. Madrid: CCS.

Navarro, Silvia (2011). De cómo Robinson Crusoe (Re) descubrió a Viernes: reflexiones sobre la perspectiva relacional de la intervención social hoy. Observatorio del Tercer sector de Bizkaia. Seminario «La perspectiva relacional de intervención». Recuperado de: http://www.3sbizkaia.org/Archivos/Documentos/Enlaces/1153_Ponencia\%20Silvia\%20Navarro\%20Pedre\%C3 $\%$ B1o.pdf

Pastor, Enrique (2004). Trabajo social comunitario. Murcia: Diego Martín Librero Editor.

Royo, Isabel, Sánchez,Susana, Lacomba, Joan, Marí, Elvira y Benlloch, Cristina. (2012). Trabajo social comunitario y desarrollo competencial. La experiencia docente de Inmodels Comunitats. Revista de Treball Social, 15, 95-106.

Sánchez, Gaspar, Escobar, Cristina y Andrés, Teodoro (2002). Migraciones y redes sociales. Salamanca: Ediciones Universidad de Salamanca.

Sánchez, Sera (2013).Un estiu al núvol. Quaderns d'educació social, 14, 44-55.

Saraceno, Benedetto (2008). Cuatro dilemas en salud mental . En: Josep $\mathrm{M}^{\mathrm{a}}$ Comelles, y Mariola Bernal, Salud mental, diversidad y cultura (pp.17-27). Madrid: Asociación Española de Neuropsiquiatría.

Sartori, Giovanni (2002). Homo videns. La sociedad teledirigida. Madrid: Santillana Ediciones generales.

Touraine, Alain (1993). Crítica de la modernidad. Madrid: Colección Temas de Hoy.

Touraine, Alain (2009). La mirada social. Un marco de pensamiento distinto para el siglo XXI. Barcelona: Editorial Paidós.

Vecina, Carlos y Ballester, Lluís (2012). Organización social, trabajo en red y desarrollo comunitario. El caso de Son Gotlen y Pere Garau. Cuadernos de Trabajo Social, 25(2), 403-412.

Verde, Carmen (2008). La Col.legi oficial de Treball Social de Catalunya.exigencia de renovación del trabajo social en contextos postbienestaristas. Revista de Treball Social, 184, 45-56.

Villalba, Cristina (1993). Redes sociales: un concepto con importantes implicaciones en la intervención comunitaria. Revista Intervención psicosocial, 2 (4), 69-85.

Villasante, Tomás (1999). Cuatro redes para hacer transformaciones sustentables. Revista Politica y Sociedad, 3, 37-54.

Wolton, Dominique (2000). Internet i després?. Una teoría crítica sobre els nous media. Barcelona: Editorial Pórtic. 


\section{Webgrafia}

http://eleducadorsocialenalaska.blogspot.com.es/

http://www.tecnolongia.org/

https://twitter.com/jaume_albaiges

http://blog.transit.es/lanza-mpc/

http://www.tecnolongia.org/?p=1361\&lang=es 